\title{
BMJ Open Enablers and barriers to primary healthcare for Aboriginal and Torres Strait Islander adolescents: study protocol for participatory mixed- methods research that builds on WHO global standards
}

Tirritpa Ritchie, ${ }^{1}$ Tara Purcell, ${ }^{2,3}$ Seth Westhead, ${ }^{1}$ Mark Wenitong, ${ }^{4,5}$ Yvonne Cadet-James, ${ }^{4,5}$ Alex Brown, ${ }^{1,6}$ Renae Kirkham, ${ }^{7}$ Johanna Neville, ${ }^{4}$ Clara Saleh, ${ }^{4}$ Ngiare Brown, ${ }^{8}$ Elissa C Kennedy, ${ }^{2}$ Julie Hennegan, ${ }^{2}$ Odette Pearson, ${ }^{1}$ Peter S Azzopardi (i) ${ }^{1,2}$

To cite: Ritchie T, Purcell T, Westhead S, et al. Enablers and barriers to primary healthcare for Aboriginal and Torres Strait Islander adolescents: study protocol for participatory mixed-methods research that builds on WHO global standards. BMJ Open 2021;11:e046459. doi:10.1136/ bmjopen-2020-046459

- Prepublication history and additional online supplemental material for this paper are available online. To view these files, please visit the journal online (http://dx.doi.org/10. 1136/bmjopen-2020-046459).

TR and TP are joint first authors. OP and PSA are joint senior authors.

Received 03 November 2020 Revised 06 April 2021 Accepted 13 April 2021

Check for updates

(C) Author(s) (or their employer(s)) 2021. Re-use permitted under CC BY-NC. No commercial re-use. See rights and permissions. Published by BMJ.

For numbered affiliations see end of article.

Correspondence to Dr Peter S Azzopardi; peter.azzopardi@sahmri.com

\section{ABSTRACT}

Introduction One-third of Australia's Aboriginal and Torres Strait Islander population are adolescents. Recent data highlight their health needs are substantial and poorly met by existing services. To design effective models of primary healthcare, we need to understand the enablers and barriers to care for Aboriginal and Torres Strait Islander adolescents, the focus of this study.

Methods and analysis This protocol was codesigned with Apunipima Cape York Health Council that supports the delivery of primary healthcare for 11 communities in Far North Queensland. We framed our study around the WHO global standards for high-quality health services for adolescents, adding an additional standard around culturally safe care. The study is participatory and mixed methods in design and builds on the recommended WHO assessment tools. Formative qualitative research with young people and their communities (exploring concepts in the WHO recommended quantitative surveys) seeks to understand demand-side enablers and barriers to care, as well as preferences for an enhanced response. Supply-side enablers and barriers will be explored through: a retrospective audit of clinic data (to identify current reasons for access and what can be strengthened); an objective assessment of the adolescent friendliness of clinical spaces; anonymous feedback from adolescent clients around quality of care received and what can be improved; and surveys and qualitative interviews with health providers to understand their perspectives and needs to provide enhanced care. This codesigned project has been approved by Apunipima Cape York Health Council and Far North Queensland Human Research Ethics Committee.

Dissemination and implications The findings from this project will inform a codesigned accessible and responsive model of primary healthcare for Aboriginal and Torres Strait Islander adolescents.

\section{Strengths and limitations of this study}

- Codesigned in partnership with the Apunipima Cape York Health Council to ensure the project is relevant, feasible, builds capacity, conducted in a culturally safe way and is translatable to action.

- Adaptation of WHO guidelines and tools (global standards) for use with Indigenous adolescents in a high-income nation, including development of items relating to culturally safe care.

- Incorporates an assessment of both demand and supply-side enablers and barriers to adolescent friendly primary healthcare, both essential considerations in strengthening models of care.

- Will contribute to an otherwise sparse literature around responsive primary healthcare for Aboriginal and Torres Strait Islander adolescents.

- Generalisability of findings to other settings may be limited, however, the process detailed is broadly generalisable.

\section{INTRODUCTION}

One-third of the Australian Aboriginal and Torres Strait Islander population are aged 10-24 years; these adolescents central to assuring the prosperity and cultural continuity of Australia's First People. ${ }^{1}$ However, as highlighted by two recent publications, Aboriginal and Torres Strait Islander adolescents have substantial health needs that are unmet by current services. ${ }^{2}{ }^{3}$ In summary, Aboriginal and Torres Strait Islander adolescents experience a heavy burden of mental disorders, suicide and self-harm, sexually transmitted infection, and injury (health conditions typical of adolescence); an excess 
burden of pneumonia and skin infections (more typical of childhood); an early onset of type 2 diabetes (more typical of adulthood); and a high burden of rheumatic heart disease and bronchiectasis (otherwise rare in Australia). ${ }^{2}$ This profile is underpinned by distinct risk exposures and determinants of health, including racism, discrimination and intergenerational trauma. As a result, adolescence is where inequities in indicators of health and well-being (such as mortality) widen between Aboriginal and Torres Strait Islander and non-Indigenous Australians. ${ }^{2}$ Adolescence also presents a substantial opportunity for health gain; more than $80 \%$ of mortality among Aboriginal and Torres Strait Islander adolescents is potentially avoidable within the current health system; these avoidable deaths are amenable to preventative interventions (rather than treatment), highlighting the need to strengthen primary healthcare. $^{2}$

Australia's health system largely provides an enabling environment for accessible primary healthcare, particularly through the Medicare universal health coverage scheme that eliminates many of the financial barriers to access. This scheme includes adolescents, with Medicare being accessible independently from age 15 years and Australian law recognising the right of mature minors to provide their own consent for healthcare. There are also specific provisions to enable access to primary healthcare for Aboriginal and Torres Strait Islander people, including through the Medicare Benefits Scheme ' 715 item' that funds an annual well person's check to facilitate health screening and promotion. However, despite these provisions, coverage of health checks in 2016 was only $22 \%$ for Aboriginal and Torres Strait Islander 15-24years old, the lowest of any age group ${ }^{4}$ and arguably at a stage of life where the opportunities for health screening are greatest. Our team is currently undertaking a systematic review (led by TP) to understand the enablers and barriers to primary healthcare for Indigenous and First Nations adolescents in Australia, New Zealand, USA and Canada. Data for Australia are limited, with evidence from other settings highlighting that Indigenous adolescents experience barriers common to all adolescents (including accessibility, concerns around consent and confidentiality), ${ }^{5}$ compounded by the specific issues including those related to racism and cultural security. ${ }^{67}$

Improving primary healthcare for adolescents is a recognised priority globally. ${ }^{8}$ Reasons for adolescents not accessing healthcare can be largely framed as those relating to: demand for services (factors at individual, household or community level that prevent access to services, including knowledge of services, sociocultural norms that limit access or services not being seen as 'relevant' to need); and supply (factors inherent to the health system that prevent service uptake, including both physical resources and competencies/skills to provide quality care). To help address these broad barriers, WHO has defined eight global standards that support adolescent's demand for primary healthcare services and the delivery of quality care (table 1). Accompanying these standards

Table 1 Global standards for quality healthcare for adolescents (reproduced from WHO, 2015) ${ }^{14}$

\section{WHO standard}

Standard 1. The health facility implements systems to ensure that adolescents are knowledgeable about their own health, and they know where and when to obtain health services.

Standard 2. The health facility implements systems to ensure that parents, guardians and other community members and community organisations recognise the value of providing health services to adolescents and support such provision and the utilisation of services by adolescents.

Standard 3. The health facility provides a package of information, counselling, diagnostic, treatment and care services that fulfils the needs of all adolescents. Services are provided in the facility and through referral linkages and outreach.

Standard 4. Healthcare providers demonstrate the technical competence required to provide effective health services to adolescents. Both healthcare providers and support staff respect, protect and fulfil adolescents' rights to information, privacy, confidentiality, non-discrimination, non-judgemental attitude and respect.

\section{Standard 5 . The health facility has convenient operating hours, a welcoming and clean} environment and maintains privacy and confidentiality. It has the equipment, medicines, supplies and technology needed to ensure effective service provision to adolescents.

Standard 6 . The health facility provides quality services to all adolescents irrespective of their ability to pay, age, sex, marital status, education level, ethnic origin, sexual orientation or other characteristics.

Standard 7. The health facility collects, analyses and uses data on service utilisation and quality of care, disaggregated by age and sex, to support quality improvement. Health facility staff are supported to participate in continuous quality improvement.

Standard 8. Adolescents are involved in the planning, monitoring and evaluation of health services and in decisions regarding their own care, as well as in certain appropriate aspects of service provision.

\section{Key concept}

Adolescent health literacy (demand)

Community support (demand)

Appropriate package of services (supply)

Providers' competencies (supply)

Facility characteristics (supply)

Equity and nondiscrimination (supply)

Data and quality improvement (demand)

Adolescents' participation (demand) 
are tools that can be used to understand supply and demand side barriers, essential to informing locally relevant responses and models of care. However, these tools have largely been developed for use in low-income and middle-income settings, and to our knowledge, not yet adapted or used with Indigenous or First Nations adolescents in high income contexts.

In this protocol, we adapt the WHO global standards and tools to explore the enablers and barriers to primary healthcare for Aboriginal and Torres Strait Islander adolescents, from both the perspectives of demand and supply. This new knowledge will be used to codesign an improved model of care for Aboriginal and Torres Strait Islander adolescents.

\section{METHODS AND ANALYSIS}

\section{Community partnership and codesign of study objectives and} research plan

This project was designed in partnership with Apunipima Cape York Health Council (Apunipima), ${ }^{9}$ the peak body for Aboriginal community-controlled primary healthcare in Australia's Far North Queensland. Apunipima supports each primary healthcare service in 11 remote Indigenous communities in Cape York and is also the primary provider of additional support programmes. Initial invitation for collaboration came in 2018 when a publication documenting health needs of Indigenous adolescents in Australia (authored by PSA, NB and AB) ${ }^{2}$ was shared with an established network of Aboriginal Community Controlled Health Organisations (MW represented Apunipima on that network). In 2019 TR, TP and PSA were invited to Apunipima to meet with clinical staff, discuss findings from previous research and consider a project together to strengthen primary healthcare for Indigenous adolescents in the Cape York. As a result, the following objectives for a research project were defined:

Objective 1: To understand the strengths, needs and preferences of Aboriginal and Torres Strait Islander young people with respect to primary healthcare (demand side). Specifically,

1. The health needs and priorities for Aboriginal and Torres Strait Islander adolescents.

2. What Aboriginal and Torres Strait Islander adolescents identify as barriers and enablers to primary healthcare.

3. What Aboriginal and Torres Strait Islander adolescents identify as key things that could be done to make primary healthcare more accessible and responsive to their health and well-being needs.

Objective 2: To understand the strengths and needs of health services and providers to deliver responsive primary healthcare for Aboriginal and Torres Strait Islander adolescents (Supply side). Specifically:

1. How often, and why, do Aboriginal and Torres Strait Islander adolescents currently access primary healthcare (identifying opportunities to strengthen existing care).
2. How does the physical environment of existing clinics align with global standards for adolescent responsive healthcare.

3. What do health providers currently provide for young people, what is their current knowledge of adolescent health, and what do they identify as training needs specific to adolescent health.

These objectives were defined to respond to local issues and needs, and also to align with global standards for quality healthcare (table 1). All eight WHO standards were considered as relevant to the provision of high quality and responsive care for Aboriginal and Torres Strait Islander adolescents in Cape York, with an additional standard (referred here as standard 9) around cultural safety also considered in developing the research tools. ${ }^{10}$ All elements of the project design (detailed below) were codesigned by the research team and Apunipima. To ensure meaningful partnership and codesign across this project we will adopt a Participatory Action Research (PAR) approach. ${ }^{11}$ PAR enables power to be shared between the participating communities and the research team, and its iterative approach of data collection and reflection is focused on developing actions which, in this case, are to strengthen primary healthcare.

\section{Target populations and research advisory group Populations}

The focus of this research is Aboriginal and Torres Strait Islander adolescents, with a specific focus on those aged 16-18 years. The age of 16 years marks an important transition in terms of health needs, capacity to provide consent and capacity to explore complex issues in research. ${ }^{12}{ }^{13} \mathrm{By}$ age 18 years, many young people also complete secondary education and transition out of communities. ${ }^{2}$ The dynamic nature of health needs across adolescence also influenced the decision for a more narrow focus on this age band for the majority of research activities, as did the advice from Apunipima that for this particular age group services need to be strengthened. In addition to young people, we will also engage parents and carers, Aboriginal and Torres Strait Islander Elders, community members and health service providers given they all contribute to the health and well-being of young people and the services they can access. With respect to communities, research efforts will be focused on three of the 11 communities Apunipima serves so as to ensure feasibility. The community partners are to be purposively selected by Apunipima taking into consideration: competing demands on the community and/or service; community priorities and readiness to focus on adolescent health and ability of the research team to travel to the community (relating to seasonal access). Findings from these three communities will be used to inform a scalable model across all 11 communities and beyond.

\section{Advisory group}

An advisory group will be established to ensure research is aligned with needs, meaningful data is generated, 
Table 2 Summary of study design for the objectives of the study

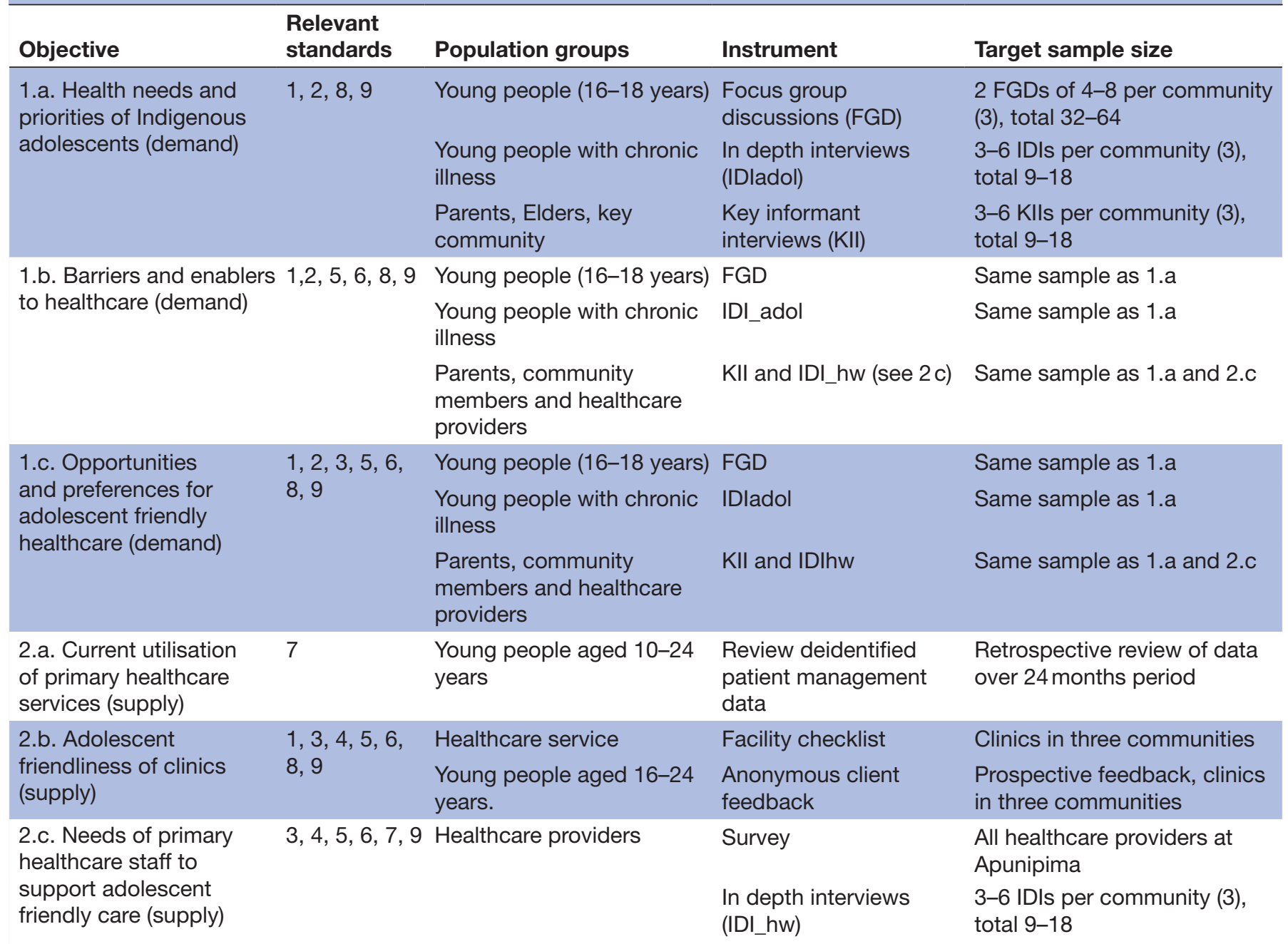

For each objective (and relevant standards, table 1), this table summarises the population groups, design and target sample. Instruments are shown in table 3.

interpretation is contextualised, outcomes are translatable and inclusive of existing and building local capacity. The advisory group of approximately 10-15 members will include core members of Apunipima as well as members of the communities where the research will occur. We will aim for involvement of young people (aged 16-24 years and diverse in gender, engagement with services and health needs), service providers, Elders and community members. Consideration will be given as to whether or not this format is conducive of meaningful engagement of the younger members and adjusted accordingly. This advisory group will inform implementation of research activities, interpretation of findings, framing of recommendations and informing important next steps.

\section{Data collection instruments}

Data collection instruments for this study include qualitative focus groups and interviews (particularly to explore the demand side) and quantitative questionnaires, facility checklists and an audit of clinic data to understand the supply side, summarised in table 2.
To inform the development of these instruments we first mapped all eight standards and their specific criteria as defined by WHO. ${ }^{14}$ We added a ninth standard on cultural safety, defining specific criteria by reviewing: The National Safety and Quality Health Service Standards User Guide for Aboriginal and Torres Strait Islander Health; The Cultural Respect Framework 2016-2026 for Aboriginal and Torres Strait Islander health; and The Queensland Health Aboriginal and Torres Strait Islander Cultural Capability Framework 2010-2033. Criteria defined for this standard included: Organisational commitment to cultural safety and rights; Indigenous governance and leadership, including policies that enable this; meaningful participation of community; ensuring and supporting Indigenous workforce; ensuring a culturally welcoming environment; availability of cultural resources; and communication and service provision that is culturally sensitive.

Against the nine standards and criteria, we then mapped the specific items of the surveys and instruments 
defined by WHO. ${ }^{14-16}$ Two investigators (TR and PSA) then independently reviewed each item, removing those not considered relevant to Aboriginal and Torres Strait Islander adolescents in Australia (eg, items relating to the control of Malaria), using a comprehensive synthesis of population data as a reference. ${ }^{2}$ Where there were multiple items measuring the same construct, we reviewed and selected the most relevant item and instrument to measure the construct of interest with the aim of streamlining the instruments where possible and minimising respondent burden. This mapping was then used to draft instruments for this study. One key modification was that we developed qualitative instruments (focus group discussions (FGD) and in-depth interviews) reflecting the key concepts in the WHO surveys so as to gather formative data around needs and preferences of adolescents, community stakeholders and providers. This was because the WHO instruments have been developed for adolescents in low-income and middle-income settings and may not be sensitive to the specific needs of Indigenous adolescents. The data collection instruments were then reviewed by the investigator group and core members of the advisory group from Apunipima; prior to implementation in community these question guides will also be reviewed by advisory group members from community and adapted as necessary. These instruments developed are summarised in table 3 and shown in online supplemental appendix.

\section{Focus group discussions}

FGDs are to be had with adolescents to understand their health needs and preferences, barriers and enablers to accessing primary healthcare, and opportunities and preferences to strengthen adolescent friendly healthcare (Objective $1 \mathrm{a}-\mathrm{c}$ ). In each community, two FGDs (one for males and one for females aged 16-18 years) including 4-8 participants will be undertaken. FGDs will be guided by a semistructured interview guide, with participants encouraged to talk about broader issues and not just their own personal lived experience. Each FGD will commence with participants describing health of Indigenous young people-in terms of strengths and challenges. To facilitate the discussion, the participatory visual method of body mapping will be used. Participants will be invited to draw around another participant to create a human outline. Participants will then be invited to draw pictures, symbols or words to reflect their views around health and well-being strengths and challenges for young people in their community. This method has successfully been used about health with Indigenous young people. ${ }^{17}$ Barriers and enablers to healthcare access will then be explored. From the barriers described, the group will be invited to nominate (up to) 10 of the most important challenges for young people accessing primary healthcare. A modified priority ranking activity will engage participants to identify the barriers that they feel are the most important using sticky dots. The discussion will then move onto participants designing an ideal health service. To facilitate the discussion, the participatory visual method of community mapping will be used. ${ }^{18}$ Participants will be invited to draw pictures, symbols or words to reflect their opinions on what an ideal youth friendly service looks like. The group will be encouraged to consider what the building looks like, describe features inside the health service and enablers in the community that can support accessible primary healthcare for young people. FGDs will be audiorecorded and researchers will take handwritten notes during the sessions. Participants will be encouraged not to use names or identifying information, however if this occurs, this information will be removed at the analysis stage.

\section{In-depth interviews with adolescents living with chronic illness (IDI_adol)}

In-depth interviews with adolescents living with chronic illness (IDI_adol) will be used to augment the FGDs. We anticipate 3-6 IDIs in each of the three communities. We will focus on young people living with rheumatic heart disease and type 2 diabetes given these conditions are common in the partner communities and these young people are likely high utilisers of primary healthcare. These interviews will explore similar concepts to the FGDs but focus directly on the lived experiences of participants.

Key informant interviews with parents, Aboriginal and Torres Strait Islander Elders and key community stakeholders

Key informant interviews (KIIs) with parents, Aboriginal and Torres Strait Islander Elders and key community stakeholders (KIIs) will augment the perceptions of young people. Their views are especially important as these stakeholders can support adolescents seeking primary healthcare, but can also be barriers or gatekeepers to adolescents accessing the care they need. We anticipate the need for 3-6 in depth interviews in each of the three communities. These KIIs follow a similar form to the IDIs but will enable an exploration of broader social and structural enablers and barriers to care.

\section{Review of deidentified patient management data}

Review of deidentified patient management data over a 24-month period will determine the number of adolescents accessing primary health services and the key primary presenting issues; this data is key to understanding what can be strengthened. These data will be obtained from the electronic patient management software (Communicare, Telstra Health) and include age (in single year for 10-24years old), gender, clinic accessed, principle presenting reason and whether this presentation was part of a well person's check (715 MBS item billed). To place this data in context, the total number of presentations (by age in 5-year age bands and gender across clinics) will also be extracted. This analysis will be across the 11 communities that Apunipima serves.

\section{Objective facility checklist}

Objective facility checklist will be used to assess the adolescent friendliness of the clinic with respect to physical 
Table 3 Study instruments

\begin{tabular}{|c|c|c|c|}
\hline Study instrument & WHO tool & Key adaptations & Concepts measured in study instrument \\
\hline $\begin{array}{l}\text { FGD: Semistructured } \\
\text { focus group } \\
\text { discussions utilising } \\
\text { participatory methods: } \\
\text { body mapping, priority } \\
\text { ranking and service } \\
\text { mapping. }\end{array}$ & $\begin{array}{l}\text { Adolescent in } \\
\text { the community } \\
\text { interview tool (quant } \\
\text { survey) }\end{array}$ & $\begin{array}{l}\text { Original quantitative tool } \\
\text { was developed into a } \\
\text { qualitative instrument to } \\
\text { gather rich formative data. }\end{array}$ & $\begin{array}{l}\text { Strengths (what keeps you strong) and challenges } \\
\text { (main problems and concerns); enablers and } \\
\text { barriers to accessing primary healthcare; and } \\
\text { opportunities to strengthen services (ideal service } \\
\text { design, what services does it provide, skills of } \\
\text { providers). }\end{array}$ \\
\hline
\end{tabular}

IDI adol:

Semistructured indepth interviews with young people with chronic illness

KII: Semistructured key informant interviews community members.

Review of deidentified N/A N/A

patient management data

\begin{tabular}{|c|c|c|}
\hline Facility checklist & $\begin{array}{l}\text { Observation tool } \\
\text { and facility checklist } \\
\text { (16 items) }\end{array}$ & $\begin{array}{l}\text { Instrument largely } \\
\text { maintained as } \\
\text { recommended by WHO, } \\
\text { with additional items } \\
\text { included to capture } \\
\text { cultural safety. }\end{array}$ \\
\hline $\begin{array}{l}\text { Anonymous client } \\
\text { feedback. To be } \\
\text { self-completed and } \\
\text { deposited following } \\
\text { clinical service. }\end{array}$ & $\begin{array}{l}\text { Adolescent client } \\
\text { exit interview tool } \\
\text { (Survey) } \\
34 \text { questions }\end{array}$ & $\begin{array}{l}\text { Adapted WHO tool to a } \\
\text { simple survey (including } \\
\text { visual rating scales) that } \\
\text { can be self-completed for } \\
\text { feasibility. }\end{array}$ \\
\hline Health provider survey & $\begin{array}{l}\text { Healthcare provider } \\
\text { interview tool } \\
\text { (Survey) } \\
35 \text { items }\end{array}$ & $\begin{array}{l}\text { Core content maintained, } \\
\text { adapted to include larger } \\
\text { emphasis on current } \\
\text { practices and needs } \\
\text { around support and } \\
\text { training (so as to inform a } \\
\text { potential response). }\end{array}$ \\
\hline $\begin{array}{l}\text { IDIhw: Semistructured } \\
\text { Key informant } \\
\text { interviews with } \\
\text { healthcare providers }\end{array}$ & $\begin{array}{l}\text { Based on health } \\
\text { provider survey (as } \\
\text { above) }\end{array}$ & $\begin{array}{l}\text { Adapted from quantitative } \\
\text { survey so as to generate } \\
\text { rich formative data. }\end{array}$ \\
\hline
\end{tabular}

As above

As above. These IDIs are As above but focussing on the lived experiences of focused around the lived young people with chronic illness who are likely high experiences as opposed to users of primary care.

FGDs above that explore issues broadly.

\section{Adult in community Adapted from quantitative Perceived strengths and challenges for} survey so as to generate rich formative data.

young people; enablers and barriers to young people accessing primary healthcare services; opportunities to strengthen care.

Retrospective audit (24 months) of clinic data. Key indicators include: age, gender, clinic being accessed, principle reason for the person's presentation, and whether this presentation was part of a well person's check (715 MBS item billed).

Facility operating hours, waiting area set up and information (including cultural relevance), availability of key medicines and equipment, client privacy and confidentiality, guidelines and decision support tools.

Age and gender, what services provided (including elements of psychosocial assessment), satisfaction with services including cultural safety of those services, opportunities to improve service provision.

Current role, reasons for having seen adolescents in clinic, current services provided when seeing young people (including psychosocial screening), knowledge around adolescent care and legislation, use of guidelines and tools, needs around training and support, and recommendations to improve care to adolescents.

Perceived health issues for young people, enablers and barriers for young people well-being and services access, service delivery, opportunities to strengthen care (with focus on supply side).

This table shows the study instruments, their adaptation from WHO tools and concepts measured. Instruments are provided in the online supplemental appendix.

FGD, focus group discussion; N/A, not available.

environment, resources, policies and procedures. This assessment will be conducted in the three communities and largely use the tool as defined by WHO, modified to include assessment of cultural safety.

Anonymous client survey following primary health service

Anonymous client survey following primary health service will enable a prospective assessment of the quality of care provided, and opportunities to improve that care.
The original WHO tool is a formal interviewer-assisted survey of considerable length. We adapted this to be brief (two pages) with visual rating scales and opportunities to provide written feedback. We also adapted this tool to be self-completed for feasibility, but also to minimise response bias. Following a clinical consultation with a young person aged 16-24 years, the healthcare provider will invite the young person to anonymously complete 
the feedback and deposit it in a locked box in the clinic; posters in the waiting area will also advertise this opportunity to provide feedback. This approach enables only those of eligible age to provide feedback. Administrative data on attendance provides a denominator to calculate completion rate.

\section{Health provider survey}

Health provider survey will explore current knowledge and practices with respect to adolescent primary care and identify areas of need with respect to support and training. All primary healthcare providers across all 11 communities will be invited to complete this survey.

\section{In-depth interviews with health providers}

In-depth interviews with health providers (IDI_hw) will further explore views and perspectives about young people accessing health services, barriers to healthcare, and how health services can be improved, with a focus on supply side. KIIs will be audio recorded and notes taken.

\section{Design adaptations due to the COVID-19 pandemic}

The COVID-19 pandemic has resulted in restricted of domestic travel in Australia, with travel to remote Indigenous communities largely closed. While we ideally would have sequenced the research to begin with qualitative work in communities to understand demand, we have adapted our design to commence with first exploring the supply side enablers and barriers. On the advice of Apunipima we will first deploy (March 2021) the health provider questionnaire for all health staff. We have adapted the health provider survey to be completed online and will also explore the potential of conducting the in-depth interviews with health providers online. We will also review routinely collected administrative data across all 11 communities; on reflection this sequencing may help identify communities to invite to partner in the research which we plan for early 2021 once travel is possible. Changes have also occurred with regard to the advisory group. While the advisory group will eventually include representation from partner communities, in the first instance the advisory group includes young people who are staff of Apunipima and an established youth advisory group (Deadly Indigenous Youth Doing Good) external to Apunipima.

\section{Sample size}

All primary healthcare providers (Aboriginal and Torres Strait Islander health workers, youth workers, nurses, doctors, allied staff) working across the 11 communities that Apunipima serves will be invited to complete the health provider survey. The majority of other components of the study are qualitative, and we have estimated the number of participants taking into consideration diversity of the sample and feasibility. During qualitative data collection, the concept of 'saturation' will be used to assess if additional data needs to be collected to satisfy the aims of the study. If so, more participants will be recruited.

\section{Participant recruitment}

Recruitment of health providers to the research will be facilitated by the Health Action Team at Apunipima; identity of staff will not be collected. Recruitment of young people and their community in the three communities will be codesigned with the advisory group once the communities have been selected and agreed to participate. Potential approaches include advertisement and invitation to participate through posters at the health clinic and local media (including social media), augmented by purposive sampling of young people with diverse experiences and needs as identified by youth and community leaders. The locations for the qualitative data collection will be discussed with the advisory group and will be at a mutually agreed safe place, which may not be the health service.

\section{Informed consent}

Parents, Elders, community members and healthcare providers over the age of 18 years will provide their own informed consent to participate (either in person or electronically). For young people aged 16-18 years, written consent for participation in the qualitative research will be obtained from parents or guardians, with written assent also obtained from all young participants. We will inform all participants that they can withdraw at any time of data collection.

For the anonymous client feedback, the healthcare provider referring the young person to complete the feedback will provide an information sheet, with consent for participation implied by the completion of anonymous feedback. This approach has been adopted so as not to burden health providers with the need to collect consent and to prevent bias. Further, young people aged 16-18 years have the capacity to understand the benefits and risks of participating in this low-risk activity. ${ }^{12} 13$

\section{Data management and security}

All paper records, including consent forms, will be stored in a locked cabinet in a secure room at Wardliparingga Aboriginal Health Equity theme at SAHMRI (Wardliparingga). Raw electronic data (including audio recordings) will be stored on password protected devices and computers at Wardliparingga. Paper records and electronic data will be securely stored for at least 7 years after collection. At the end of this period all hard copies of documents will be shredded, and electronic copies deleted. Data will only be accessible to authorised members of the research team. Deidentified and cleaned data sets will be provided to Apunipima and shared among investigators using a secure, password-protected cloud.

The only raw data to be exchanged electronically will be data collected from health providers. The questionnaire will be collected using REDCap, and other than clinical role and Indigenous status, no other identifying information will be captured. REDCap data are encrypted in transit via transport-layer security (industry best standard), with the dataset securely stored as outlined above. 


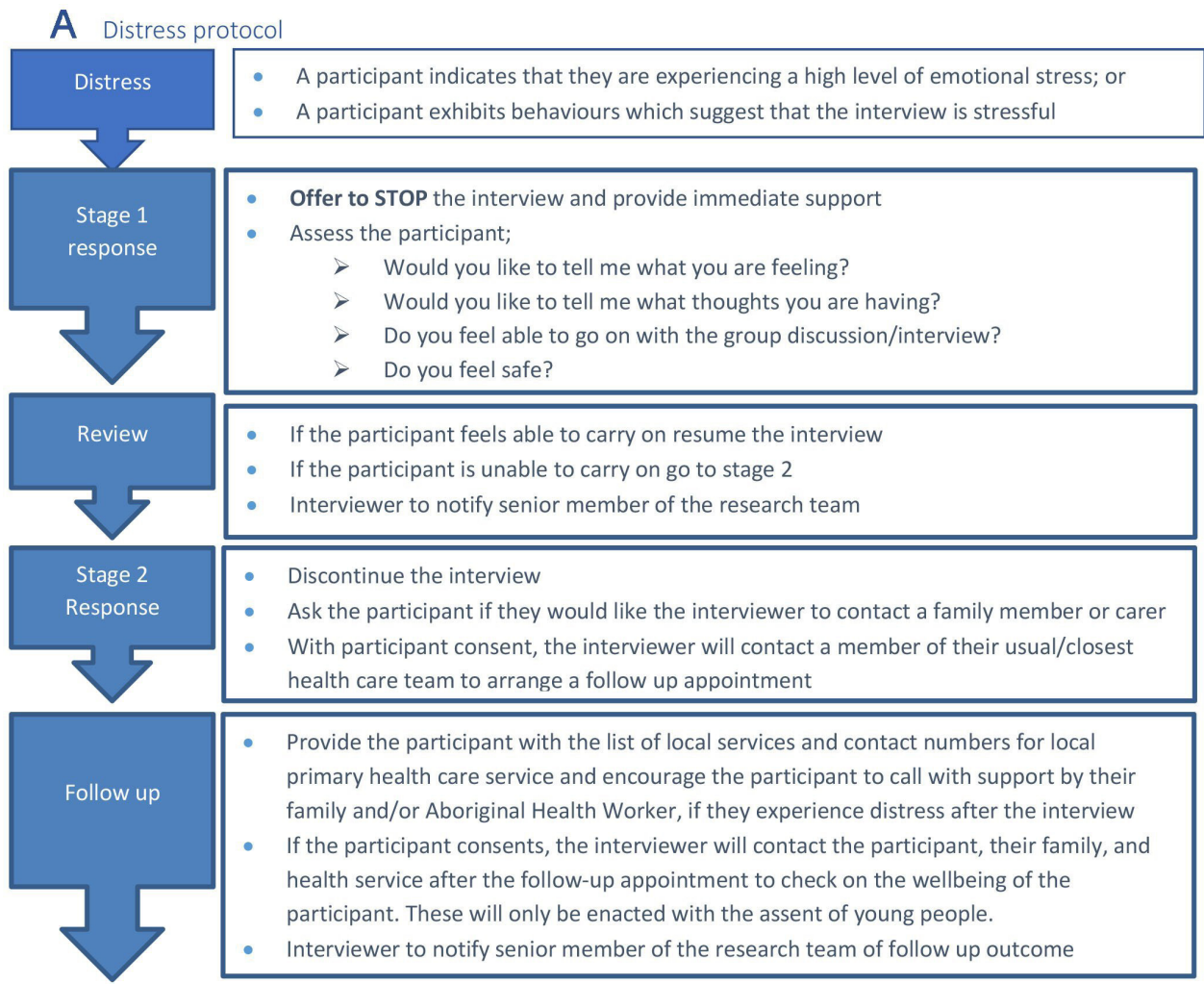

B Mandatory reporting protocol

Mandatory reporting relates to risk of abuse/ neglect / harm from others, risk of harm from self, or risk of harming others.

\begin{tabular}{|c|c|}
\hline $\begin{array}{l}\text { Beginn } \\
\text { inter }\end{array}$ & $\begin{array}{l}\text { Obtain consent from parents/guardians, and assent from young people to participate in } \\
\text { this study. As part of this consent/ assent procedure, the need to potentially breach } \\
\text { confidentiality (if a young person is at risk of harm, or harming others) is discussed. }\end{array}$ \\
\hline $\begin{array}{l}\text { e } 1 \\
\text { sse to } \\
\text { ure of }\end{array}$ & $\begin{array}{l}\text { - STOP the interview and advise participant that what they have disclosed meets } \\
\text { reasonable cause for suspicion of harm to self or others. } \\
\text { - Offer immediate support if participant is distressed } \\
\text { - Reassure the young person that your main concern is to keep them/ others safe. }\end{array}$ \\
\hline & $\begin{array}{l}\text { - Interviewer to notify senior member of the research team to assist with next steps } \\
\text { - Explain to the participant that you are notifying a family member or carer- involve them in } \\
\text { the decision around who the best person is. } \\
\text { - Notify appropriate service depending on the nature of the risk }\end{array}$ \\
\hline Follow up & $\begin{array}{l}\text { Provide the participant with the list of local services and contact numbers and encourage } \\
\text { the participant to call if they experience distress after the interview } \\
\text { - Research team of follow up outcome of the referral. }\end{array}$ \\
\hline
\end{tabular}

Figure 1 Response to distress (A) and concern around safety (B).

The in-depth interviews with healthcare providers will be conducted over Zoom videoconferencing using a password protected link, with the discussion recorded using the inbuilt recording feature and securely stored as above.

\section{Data analysis plan}

The health provider survey, facility checklist and client feedback survey will be quantitatively analysed using WHO analysis guidelines. ${ }^{19}$

Audiorecordings of interviews will be transcribed verbatim. Transcripts will be analysed by two researchers thematically using an inductive 'data-driven' process, with codes identified from the empirical material. ${ }^{20}$ Data extracts will be selected to illustrate key constructs. No personal or other identifying data (including details that could identify participating organisations or individuals) will be included in summaries or other research outputs.

Aggregated deidentified patient management data will be analysed using descriptive quantitative methods (frequencies) to report the rates of the different clinical presentations by age and gender. Population estimates from the Australian Bureau of Statistics (by age and sex) for the communities that Apunipima services will enable 
estimation of age-specific and sex-specific access rates per population denominator.

\section{Patient and public involvement}

As detailed above, this project was codesigned in partnership with Apunipima Cape York Health Council. This involved codesign of the objectives, research tools and dissemination strategy. This codesign was to ensure that the project is aligned with needs and translatable to action-it also represents best practice in Aboriginal health research. ${ }^{21}$ Further, once the focal communities for this research are selected, we will establish an advisory group that will include membership from those communities to ensure local knowledge, ownership and translation. This advisory group will be involved in the implementation of the research, however, not directly involved in the qualitative inquiry to ensure confidentiality is maintained. The advisory group will also support dissemination (detailed below). They will be formally acknowledged in all publications and materials resulting from this work.

\section{ETHICS}

\section{Ethics review}

The research protocol was first fully reviewed and endorsed by Apunipima's Research Review Panel. The project subsequently received ethics approval from Far North Queensland Human Research Ethics Committee (HREC/2019/QCH/57297, with amendment for online health provider survey AM/2020/QCH/57297).

\section{Benefits and risks}

There are no direct benefits for individuals participating in the study. However, the information provided during the project may help strengthen healthcare services to meet the health needs of adolescents. Possible risks include discomfort from talking about particular issues and disclosure of sensitive health related information that requires clinical review. This project has been designed to ensure that the risk of participants experiencing distress is low. Specifically, we will not be probing for distressing issues. To minimise risk we will exclude participants who are acutely unwell. We will also be obtaining consent from parents and assent from participants themselves. A Distress Protocol has been developed to guide the research team response to support any participants who experience distress or the need to report risk of harm (figure 1). We will also provide all participants with a follow-up card at the completion of the qualitative enquiry which will include contact numbers of the research team and also key healthcare providers. The fact that this research is being conducted in partnership with a primary healthcare provider is enabling of appropriate follow-up of those who require it.

\section{Dissemination and implications}

A final report of results will be provided to Apunipima Cape York Health Council. These will also be formally presented at dissemination workshops held at Apunipima Cape York Health Council and the three participating communities, and to other audiences as defined by the Advisory Group. In collaboration with Apunipima Cape York Health Council, data collected during this study will be published in peer reviewed journals and/or presented at a conference. The findings from this project will inform a codesigned accessible and responsive model of primary healthcare for Aboriginal and Torres Strait Islander adolescents in Far North Queensland.

The implications of this project are substantial with strengthened primary care for young people having the potential to improve population health and reduce health inequities. ${ }^{1}$ Adolescents represent a third of the Aboriginal and Torres Strait Islander population, and their health needs are substantial and largely unmet. Improving health at this time of life, particularly when young people are establishing their identity, transitioning from education to employment, and developing new relationships has the potential for long-lasting impacts. Through strengthened primary care there is also the potential to identify and address health risks that typically emerge during adolescence, including obesity and risky substance use that determine non-communicable diseases in adult life, key drivers of premature mortality for Indigenous Australians. ${ }^{22}{ }^{23}$ There is also the potential to strengthen healthcare when young people may be starting to have children, assuring the best start to life for the next generation.

\section{Author affiliations}

${ }^{1}$ Wardliparingga Aboriginal Health Equity Theme, South Australian Health and Medical Research Institute, Adelaide, South Australia, Australia

${ }^{2}$ Global Adolescent Health Group, Maternal Child and Adolescent Health Program, Burnet Institute, Melbourne, Victoria, Australia

${ }^{3}$ Melbourne School for Population and Global Health, The University of Melbourne, Melbourne, Victoria, Australia

${ }^{4}$ Apunipima Cape York Health Council, Cairns, Queensland, Australia

${ }^{5}$ James Cook University, Townsville, Queensland, Australia

${ }^{6}$ Faculty of Health and Medical Sciences, The University of Adelaide, Adelaide, South Australia, Australia

${ }^{7}$ Menzies School of Health Research, Casuarina, Northern Territory, Australia ${ }^{8}$ Ngaoara Aboriginal Child and Adolescent Wellbeing, Wollongong, New South Wales, Australia

Contributors The study design was led by TR and TP with the support and supervision of $A B, O P, Y C-J, M W, R K$ and PSA. SW joined the research team in early 2020 and has led the implementation of efforts since, including establishment of the study's advisory group. All authors (TR, TP, SW, MW, YC-J, AB, RK, JN, CS, $\mathrm{NB}, \mathrm{EK}, \mathrm{JH}, \mathrm{OP}$ and PSA) contributed to the drafting of the manuscript, critically reviewed its content and approved its publication.

Funding This work was supported by the National Health and Medical Research Council-funded programme 'Improving Health Outcomes in the Tropical North: a multidisciplinary collaboration (Hot North)', grant identification number 1131932.

Competing interests None declared.

Patient consent for publication Not required.

Provenance and peer review Not commissioned; externally peer reviewed.

Supplemental material This content has been supplied by the author(s). It has not been vetted by BMJ Publishing Group Limited (BMJ) and may not have been peer-reviewed. Any opinions or recommendations discussed are solely those of the author(s) and are not endorsed by BMJ. BMJ disclaims all liability and responsibility arising from any reliance placed on the content. Where the content 
includes any translated material, BMJ does not warrant the accuracy and reliability of the translations (including but not limited to local regulations, clinical guidelines, terminology, drug names and drug dosages), and is not responsible for any error and/or omissions arising from translation and adaptation or otherwise.

Open access This is an open access article distributed in accordance with the Creative Commons Attribution Non Commercial (CC BY-NC 4.0) license, which permits others to distribute, remix, adapt, build upon this work non-commercially, and license their derivative works on different terms, provided the original work is properly cited, appropriate credit is given, any changes made indicated, and the use is non-commercial. See: http://creativecommons.org/licenses/by-nc/4.0/.

\section{ORCID iD}

Peter S Azzopardi http://orcid.org/0000-0002-9280-6997

\section{REFERENCES}

1 Azzopardi P, Blow N, Purcell T. The health of first nations adolescents as the foundation to health equity. MJA 2020;212:202-4.

2 Azzopardi PS, Sawyer SM, Carlin JB, et al. Health and wellbeing of Indigenous adolescents in Australia: a systematic synthesis of population data. Lancet 2018;391:766-82.

3 Australian Institute of Health and Welfare. Aboriginal and Torres Strait Islander adolescent and youth health and wellbeing 2018. Canberra: AlHW, 2018.

4 Australian Insitute of Health and Welfare. Indigenous health check (MBS 715) data tool, 2016. Available: https://www.aihw.gov.au/ reports/indigenous-health-welfare-services/indigenous-health-checkmbs-715-data-tool/contents/dynamic-data-displays

5 Tylee A, Haller DM, Graham T, et al. Youth-friendly primary-care services: how are we doing and what more needs to be done? Lancet 2007;369:1565-73.

6 Kilian A, Williamson A. What is known about pathways to mental health care for Australian Aboriginal young people?: A narrative review. Int J Equity Health 2018;17:12.

7 Warwick S, Atkinson D, Kitaura T, et al. Young aboriginal people's perspective on access to health care in remote Australia: hearing their voices. Prog Community Health Partnersh 2019;13:171-81.

8 World Health Organization. Global standards for quality healthcare services for adolescents: a guide to implement a standardsdriven approach to improve the quality of health care services for adolescents. (Volume 1: Standards and criteria). Geneva, 2015.

9 Apunipima Cape York Health Council. Available: http://www. apunipima.org.au/about [Accessed 1 Apr 2021].
10 Coffin J. Rising to the challenge in Aboriginal health by creating cultural security. Aborig Is/ Health Work J 2001;31:22-4.

11 Baum F, MacDougall C, Smith D. Participatory action research. $J$ Epidemiol Community Health 2006;60:854-7.

12 Santelli J, Haerizadeh S, MCGowan T. Inclusion with protection: obtaining informed consent when conducting research with adolescents. In: Santelli J, Balvib N, eds. Conducting research with adolescents in low and middle income countries. Florence, Italy: UNICEF Innocenti, 2017.

13 Patton GC, Sawyer SM, Santelli JS, et al. Our future: a Lancet commission on adolescent health and wellbeing. Lancet 2016;387:2423-78

14 WHO, UNAIDS. Global standards for quality health-care services for adolescents: a guide to implement a standards-driven approach to improve the quality of health care services for adolescents. Volume 1: Standards and criteria), 2015.

15 WHO, UNAIDS. Global standards for quality health-care services for adolescents: a guide to implement a standards-driven approach to improve the quality of health care services for adolescents. Volume 3: Tools to conduct quality and coverage measurement surveys to collect data about compliance with the global standards), 2015.

16 UNAIDS, WHO. Global standards for quality health-care services for adolescents: a guide to implement a standards-driven approach to improve the quality of health care services for adolescents. Volume2: implementation guide). Geneva, 2015.

17 Chenhall R, Davison B, Fitz J, et al. Engaging youth in sexual health research: Refining a "youth friendly" method in the Northern Territory, Australia. Visual Anthropol Rev 2013;29:123-32.

18 Skinner E, Masuda JR. Right to a healthy city? Examining the relationship between urban space and health inequity by Aboriginal youth artist-activists in Winnipeg. Soc Sci Med 2013;91:210-8.

19 WHO, UNAIDS. Global standards for quality health-care services for adolescents: a guide to implement a standards-driven approach to improve the quality of health care services for adolescents. Volume 4: Scoring sheets for data analysis), 2015

20 Braun V, Clarke V. Using thematic analysis in psychology. Qual Res Psychol 2006;3:77-101.

21 NHMRC. Road map 3: a strategic framework for improving Aboriginal and Torres Strait Islander health through research. Canberra: National Health and Medical Research Council, 2018.

22 Azzopardi P, Brown AD, Zimmet P, et al. Type 2 diabetes in young Indigenous Australians in rural and remote areas: diagnosis, screening, management and prevention. Med J Aust 2012;197:32-6.

23 Vos T, Barker B, Begg S, et al. Burden of disease and injury in aboriginal and torres strait islander peoples: the indigenous health gap. Int J Epidemiol 2009;38:470-7. 